\title{
Spec-seq unveils transcriptional subpopulations of antibody-secreting cells following influenza vaccination
}

\author{
Karlynn E. Neu, ${ }^{1,2}$ Jenna J. Guthmiller, ${ }^{2}$ Min Huang, ${ }^{2}$ Jennifer La, ${ }^{3}$ Marcos C. Vieira, ${ }^{4}$ Kangchon Kim, ${ }^{4}$ Nai-Ying Zheng, ${ }^{2}$ \\ Mario Cortese, ${ }^{5}$ Micah E. Tepora, ${ }^{1}$ Natalie J. Hamel, ${ }^{1}$ Karla Thatcher Rojas, ${ }^{2}$ Carole Henry, ${ }^{2}$ Dustin Shaw, ${ }^{1,2}$ \\ Charles L. Dulberger, ${ }^{6}$ Bali Pulendran, ${ }^{5}$ Sarah Cobey, ${ }^{4}$ Aly A. Khan, ${ }^{7}$ and Patrick C. Wilson ${ }^{1,2}$
}

'The Committee on Immunology, ${ }^{2}$ The Department of Medicine, Section of Rheumatology, ${ }^{3}$ The Department of Pathology, Molecular Pathogenesis and Molecular Medicine, and ${ }^{4}$ The Department of Ecology and Evolution, The University of Chicago, Chicago, Illinois, USA. ${ }^{5}$ Emory Vaccine Center, Emory University, Atlanta, Georgia, USA. ${ }^{6}$ The Department of Biochemistry and Molecular Biophysics, The University of

Chicago, Chicago, Illinois, USA. ${ }^{\top}$ Toyota Technological Institute at Chicago, Chicago, Illinois, USA.

\begin{abstract}
Vaccines are among the most effective public health tools for combating certain infectious diseases such as influenza. The role of the humoral immune system in vaccine-induced protection is widely appreciated; however, our understanding of how antibody specificities relate to $B$ cell function remains limited due to the complexity of polyclonal antibody responses. To address this, we developed the Spec-seq framework, which allows for simultaneous monoclonal antibody (mAb) characterization and transcriptional profiling from the same single cell. Here, we present the first application of the Specseq framework, which we applied to human plasmablasts after influenza vaccination in order to characterize transcriptional differences governed by $B$ cell receptor (BCR) isotype and vaccine reactivity. Our analysis did not find evidence of long-term transcriptional specialization between plasmablasts of different isotypes. However, we did find enhanced transcriptional similarity between clonally related B cells, as well as distinct transcriptional signatures ascribed by BCR vaccine recognition. These data suggest IgC and IgA vaccine-positive plasmablasts are largely similar, whereas IgA vaccine-negative cells appear to be transcriptionally distinct from conventional, terminally differentiated, antigen-induced peripheral blood plasmablasts.
\end{abstract}

\section{Introduction}

The medical field has relied on vaccines for improving human health since the days of smallpox inoculation-long before antibodies were identified $(1,2)$. Vaccines induce a humoral immune response, in which $\mathrm{B}$ cells mediate antibody protection against a wide range of bacterial and viral pathogens, including influenza. The influenza vaccine provides annual protection for millions of people worldwide by initiating an immune response against the 4 influenza strains most likely to circulate that year. Viral antigens within the vaccine induce a global immune response that provides immediate protection through antibody-secreting cells (ASCs), while establishing a foundation for future protection within the memory B cell compartment.

The recognition of antigen by surface-bound $\mathrm{B}$ cell receptors initiates the humoral immune response. The BCR is generated through a process of recombination and somatic hypermutation of the immunoglobulin ( $\mathrm{Ig}$ ) genes, resulting in a unique receptor sequence that is only shared with the clonal progeny of that cell. The variable domains of the receptor determine the abili-

Authorship note: KEN and JJG contributed equally to this work. Conflict of interest: The authors have declared that no conflict of interest exists. License: Copyright 2019, American Society for Clinical Investigation.

Submitted: March 28, 2018; Accepted: October 9, 2018.

Reference information: J Clin Invest. 2019;129(1):93-105.

https://doi.org/10.1172/JCI121341. ty to bind certain antigens, while the Fc domain (comprised of the BCR constant domain) is essential for directing downstream immunological responses. After antigen exposure, a B cell can undergo class switch recombination (CSR), where the constant domain of the BCR is swapped. The new constant domain confers a new BCR isotype, which gives the secreted antibody distinct effector functions. These functions are tailored to best combat the current challenge, with different isotypes having different binding affinities for the C1q molecule of the complement cascade and pro- or antiinflammatory Fc receptors. Posttranslational modifications to the $\mathrm{Fc}$ domain can further alter these binding affinities and give another level of complexity to antibody regulation (3-11).

Prior to the secretion of antibodies (the soluble form of the BCR), B cells must differentiate into ASCs (the effector cells of an ongoing antibody response). Plasmablasts are the main ASCs in the peripheral blood and are thought to be terminally differentiated and short-lived $(12,13)$. These cells are present at a low level in the peripheral blood of healthy humans and undergo significant expansion 7 days after immunization or infection $(14,15)$. The vaccine-induced population is predominantly antigen-reactive and has been extensively utilized as a way to characterize the dynamics of an ongoing $B$ cell immune response $(14,16)$. This population is also a common source for therapeutic and prophylactic human mAb therapies (17). The majority of plasmablasts induced by the inactivated vaccine express the IgG 
receptor isotype (14), although some vaccine-specific cells produce IgA (18). This isotype bias is an inverse of the steady-state plasmablast population, which does not bind influenza and is primarily comprised of cells expressing the IgA receptor isotype (19). These isotype preferences exist due to the functional roles of these populations, with IgG antibodies specializing in antigen clearance through engagement with Fc receptors (20), and IgA antibodies more commonly reducing antigen immunogenicity through neutralization and sequestration (21). Although CSR alters the functional capacity of antibodies, it remains unknown if the process alters the cellular identity of the plasmablast beyond changes to the BCR transcript itself.

The steady-state IgA-secreting plasmablast population is maintained in the peripheral blood during an ongoing vaccine response (19). How these cells are related to and differ from the antigen-induced cells remains unclear. The peripheral blood steady-state plasmablasts are hypothesized to recirculate from mucosal niches (19) where they secrete antibodies that help maintain homeostasis of the microbiota (22). The recent discovery of shared IgA antibody sequences in both the serum and the gut further supports the connection between steady-state circulating IgA-secreting cells and mucosa-localized cells (23). This possible shared origin, combined with the enhanced neutralization capacity of IgA antibodies, makes them an ideal target population for vaccines aimed to induce localized mucosal protection (24-29). This current goal in vaccine development is based on the hypothesis that localized protective antibodies would prevent initial infection of the host, reducing the pathogen burden and the need for neutralizing serum IgG antibodies. Thus, further characterization of IgA plasmablasts, in particular steadystate versus antigen-reactive plasmablasts, is of great interest.

Transcriptional profiling is a powerful way to characterize cellular identity, as it gives insight into all ongoing cellular processes. Here we present Spec-seq, a framework to allow for the simultaneous exploration of the immune transcriptome, receptor repertoire, and receptor functional characteristics at the single-cell level. As a first application of this approach, we address the long-standing questions of how BCR isotype or specificity impacts the $\mathrm{B}$ cell transcriptome, and if these features direct functional specialization outside of the BCR itself. We employed Spec-seq to analyze human plasmablasts after influenza vaccination to characterize transcriptional differences dictated by BCR isotype and vaccine reactivity. Specifically, we examine how IgA vaccine-positive (IgAVP) peripheral blood plasmablasts compare with IgG vaccine-positive (IgGVP) and IgA vaccinenegative (IgAVN) populations. Our analysis reveals enhanced transcriptional similarity within clonally related plasmablasts, perhaps due to original B cell activation event imprinting. We also identified transcriptional subpopulations correlated with BCR vaccine binding. Differentially expressed genes between these populations included glycotransferase enzymes previously credited with altering the glycosylation of antibodies, thereby altering their immunogenicity, as well as proteins that regulate cell cycling. These data suggest IgGVP and IgAVP plasmablasts are largely similar, whereas IgAVN cells appear transcriptionally distinct from traditional terminally differentiated, antigeninduced peripheral blood plasmablasts.

\section{Results}

The Spec-seq framework (Supplemental Figure 1A; supplemental material available online with this article; https://doi. org/10.1172/JCI121341DS1) was developed to explore plasmablast transcriptional diversity in the context of BCR repertoire and antigen specificity. To address our specific questions of interest, IgA and $\operatorname{IgG}$ peripheral blood plasmablasts were single-cell sorted from 6 donors (Supplemental Figure 1D) 7 days after influenza vaccination (Figure $1, \mathrm{~A}$ and B). As expected with an intramuscular inactivated vaccine, there was a wide range of plasmablast expansion per donor (1.6\%-26.7\% of total B cells) (Supplemental Figure 2A), with the majority of plasmablasts expressing IgG (flow cytometry 51\%-82\%, ELISPOT 62\%-85\%) (Figure 1C). The total frequency of vaccine-binding plasmablasts per donor ranged from $48 \%-91 \%$ (Figure 1C). Monoclonal antibodies (mAbs) were cloned and generated from each plasmablast and screened by ELISA for binding to the administered vaccine and the viral components of the vaccine (Supplemental Figure 2, B-D). The mAb binding data combined with the receptor sequence information allowed each cell to be binned into 1 of our 3 groups of interest: IgAVP, IgGVP, and IgAVN (Supplemental Table 1). IgGVP mAbs had a slight increase in relative binding affinity, reported as area under the curve, compared with IgAVP mAbs (Supplemental Figure 2E). It is important to note that a minor fraction of the IgAVN cells may be vaccineinduced but were not identified as such because it is well-appreciated that it is impossible to assess all biologically derived vaccine epitopes (30). We believe the majority of these IgAVN cells are derived from the steady-state IgA plasmablast population, which is present in the peripheral blood of humans and is maintained after influenza vaccination (19). Unfortunately, the low frequency of IgG plasmablasts present in the steady-state blood and the huge induction of this population following vaccination prevented us from including a vaccine-inexperienced IgG population for comparison. These samples were then subjected to deep single-cell RNA sequencing (scRNA-seq). We focused our subsequent analyses on 291 plasmablasts that passed quality control filters (Supplemental Figure 3) with 11,895 unique genes detected in at least $5 \%$ of the cells.

The BCR isotype classification for each cell was validated on 3 levels: flow cytometry, Sanger sequencing during BCR cloning, and the BCR contig assembled from the scRNA-seq data (31) (Figure 1D). The scRNA-seq data were normalized (Supplemental Figure 3) and visualized using the t-Stochastic Neighbor Embedding algorithm (tSNE), which projects high-dimensional data into a 2-dimensional space and groups cells with similar transcriptomes together (32). As expected, after proper normalization and batch correction, clustering is not associated with experimental batch or donor (Supplemental Figure 3, H-K). Instead, a trend was revealed for the IgAVP and IgAVN cells to cluster together and away from the IgGVP population (Figure 1E), suggesting a plasmablast BCR isotype-dependent signature.

Repertoire analysis found limited differences between the 3 populations of interest, and overall did not suggest distinct cellular compartments of origin (Supplemental Figure 4 and Supplemental Tables 1 and 2). We did observe a significantly increased prevalence of the VH1 gene within the IgGVP population; how- 
A

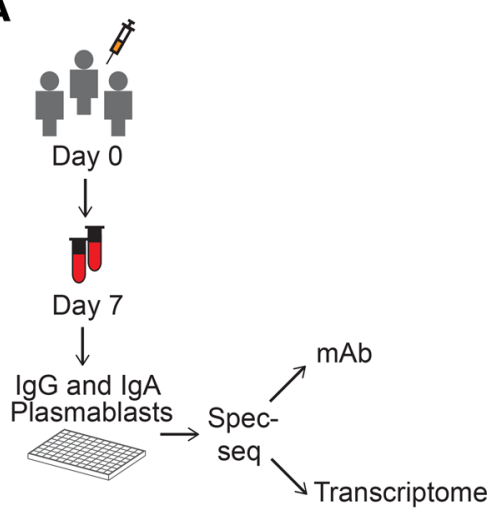

B

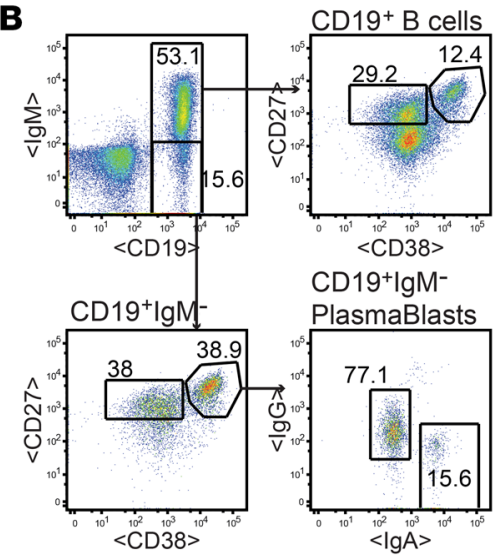

C

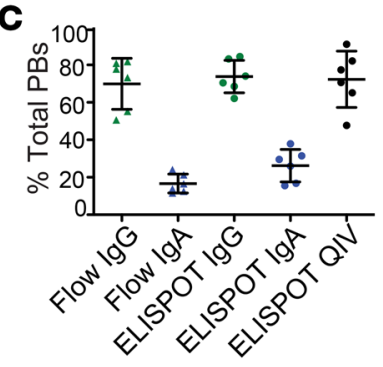

D

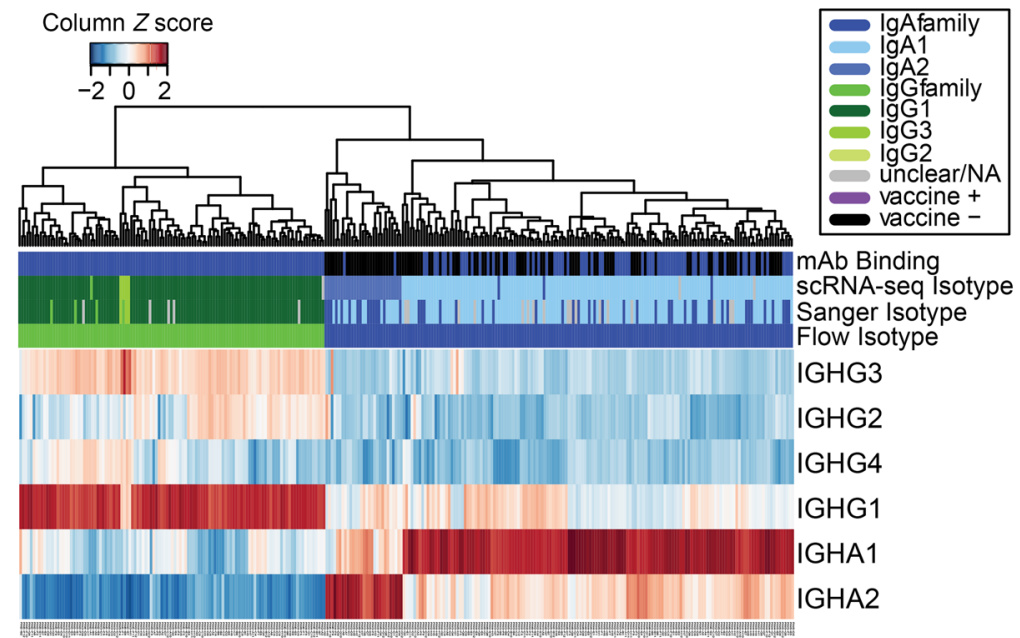

E

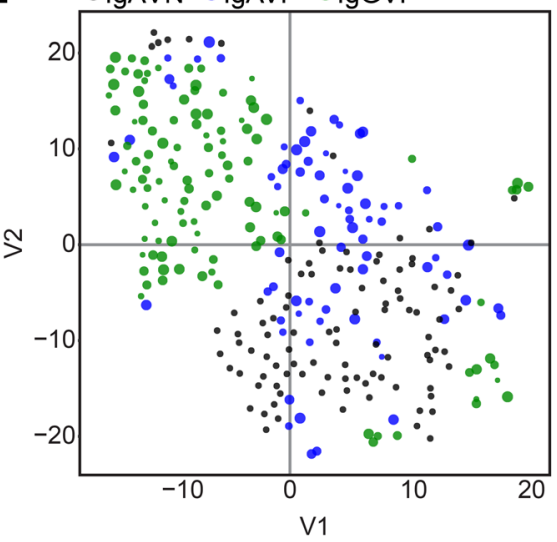

Figure 1. Experimental approach and characterization of influenza-induced plasmablast populations. (A) Experimental outline. (B) Sorting strategy. IgM- plasmablasts were identified as $\mathrm{CD} 19^{+} \mathrm{CD} 27^{++} \mathrm{CD} 38^{++}$and either $\lg \mathrm{C}^{+}$or IgA $\mathrm{A}^{+}$. (C) Frequency of total plasmablasts of each isotype as detected during cell sorting and through anti-Ig ELISPOT. The ELISPOT data also report the frequency of vaccine-specific plasmablasts (QIV). Line indicates frequency mean. (D) Heatmap of all 291 single cells, clustered by their relative expression of the 4 IgG subtype genes and the 2 lgA subtype genes. Annotation bars across the top show the mAb vaccine binding and 3 levels of BCR isotype validation. (E) tSNE projection of the entire transcriptome of all 291 cells colored by group ID and with monoclonal antibody vaccine binding area under the curve (AUC) indicated by spot size.

ever, this is difficult to interpret without repertoire from an IgGVN population. Furthermore, we also observed a substantial increase in the frequency of IgAVN cells versus IgAVP cells utilizing the IgA subtype 2 constant domain (IGHA2), which has been reported as more prevalent within mucosa-localized antibodies (33). Although it is difficult to draw conclusions from repertoire studies of this scale, the enhanced IGHA2 usage further supports a connection between the peripheral blood IgAVN population and the mucosal IgA ASC population.

Further repertoire analysis revealed evidence of clonotype expansion and identified 100 of the total 291 plasmablasts as members of 29 clonal expansions (Figure 2, A and B). The high frequency of clonal expansion is not uncommon after influenza vaccination (14). The clonal families ranged in size from 2-13 detected members and were present in all 3 populations of interest, with 3 unique vaccine-positive clones that span the IgG and IgA compartments (clones 4, 13, and 21). Within the 3 donors where these shared clones were detected, they were found at a
$10 \%-20 \%$ frequency, which is similar to what we detected with independent high-throughput repertoire sequencing studies (16.5\%-25.4\%) (Figure 2, A and C). Clonal expansions containing cells of different isotypes have been previously reported $(34,35)$, and the tendency for BCR sequences to cluster within them by isotype suggests early CSR divergence before continued affinity maturation (Figure 2B). No difference in the relative binding affinity of IgG or IgA clonal family members was observed in our data, although the significance of this analysis is limited by the number of clonal expansions identified (data not shown). This emphasizes the importance of exploring transcriptional differences between IgGVP and IgAVP plasmablasts, as it increases the possibility of separate transcriptional, or functional, identities beyond the BCR. No BCR overlap was identified between the vaccine binding plasmablasts and the IgAVN population. These data suggest that only the vaccine-binding plasmablasts share cellular ancestors, which furthers our interest in characterizing the unique qualities of the IgAVN plasmablast population. 

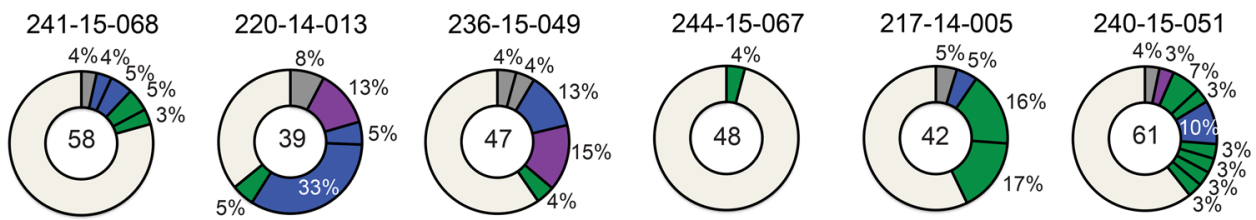

$\square \lg \mathrm{G} V+$ clon

- $\lg A \mathrm{~V}+$ clone ulgA $V$ - clone

$\square \lg G \& \lg A V+$ clone

口Not clonal

B

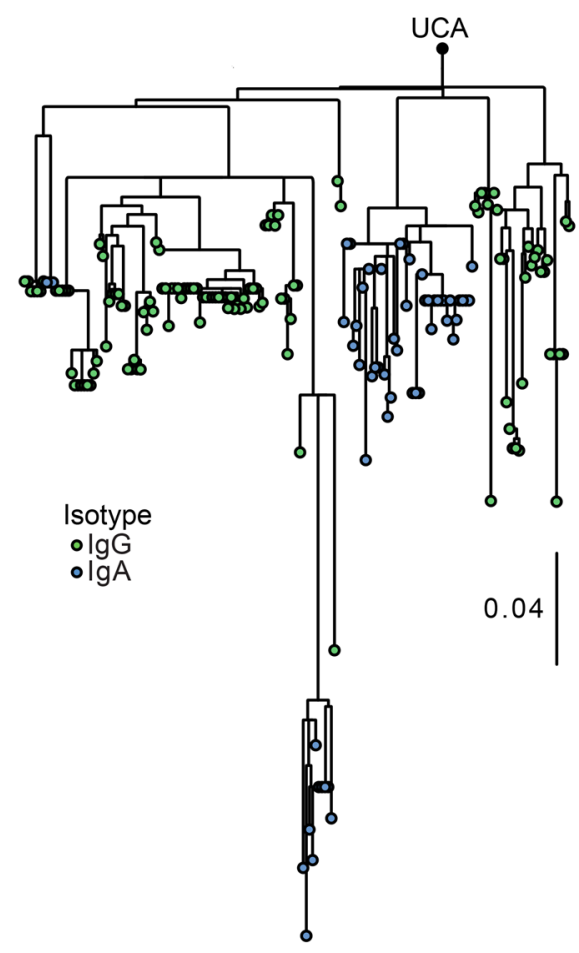

C

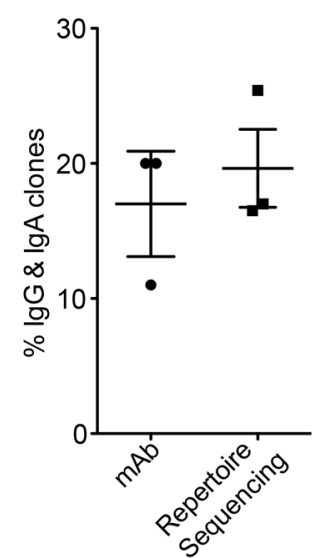

D Vaccine Positive : Vaccine Negative

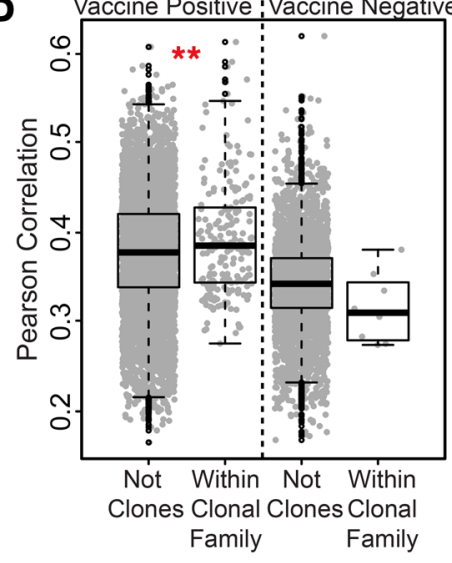

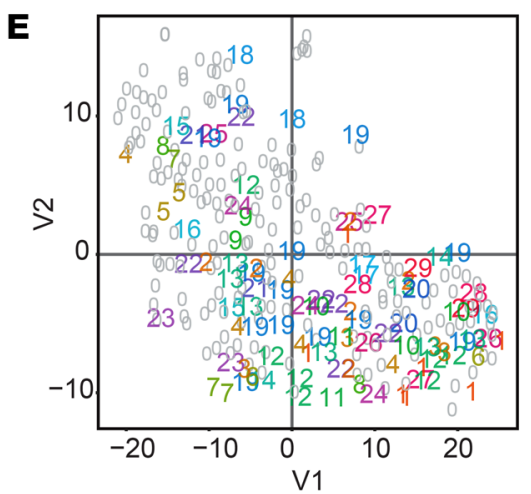

Figure 2. Clonal plasmablasts display increased transcriptional similarity. (A) Clonal expansions are indicated by donor. (B) Representative clonal tree from analysis of high-throughput repertoire sequencing data. (C) Frequency of clones containing both IgG and IgA members for the 3 donors where these clonal expansions were identified. Data mean indicated with line. (D) Pearson correlation coefficients were calculated for all pairwise comparisons of unrelated B cells and between clonal B cells within the same clonal family, for both the vaccine-positive (clonal, $n=87 ;$ not clonal, $n=108$ ) and vaccine-negative (clonal, $n=13$; not clonal, $n=87$ ) compartments after exclusion of all Ig genes. Box plots display median correlation ( ${ }^{* *} P<2 \times 10^{-7}$, Welch's 1 -sided $t$ test; Methods). (E) tSNE projection of all 3 populations $(n=295)$ after exclusion of Ig genes, with clonal families designated by numbers $1-29$, and unrelated B cells indicated by gray zeros.

The identification of clonal expansions within the IgAVN population was surprising due to the expansive combinatorial diversity of the BCR, and we have 2 potential explanations for this clonal expansion. The first is that the soluble activation signals occurring during antigen-specific plasmablast activation were sufficient for BCR-independent B cell activation and subsequent proliferation of these cells. Alternatively, the expansion of IgAVN clones could support a more restricted antigen specificity within the IgAVN population than previously expected, resulting in an overall less diverse repertoire. Both of these hypotheses require further exploration in larger clonal data sets.

This unique data set allowed us to ask if B cells from the same clonal family have transcriptional profiles that are more alike than unrelated B cells. When clonally related B cells were labeled in the
tSNE projection, clusters appeared (Supplemental Figure 5A). To quantitatively evaluate this observation, the Pearson correlation coefficient was calculated for each pair of cells in a clonal family as a way to assess overall transcriptional similarity. The average correlation between clonal family members within the vaccine-positive population is significantly higher than that of the unrelated vaccine-positive B cells (Supplemental Figure 5B), indicating increased transcriptional similarity within clonal families. This trend was not detected in the vaccine-negative IgA population (Supplemental Figure $5 B$ ). Since B cell clones share the same BCR transcripts, we repeated our analysis in the absence of Ig genes. For clonally related samples in both the vaccine-positive and vaccine-negative populations, correlations are reduced upon removal of Ig genes (Supplemental Figure 5C), whereas unrelated plasmablast correla- 
A

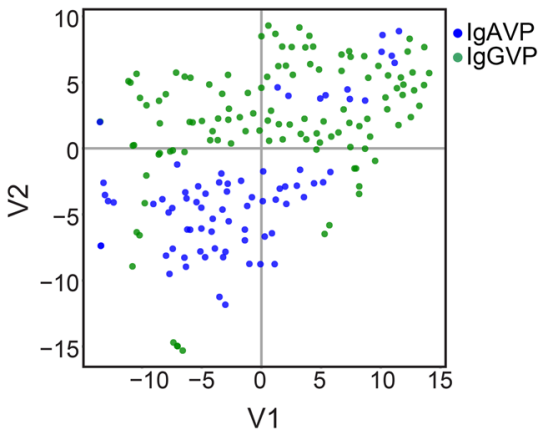

B

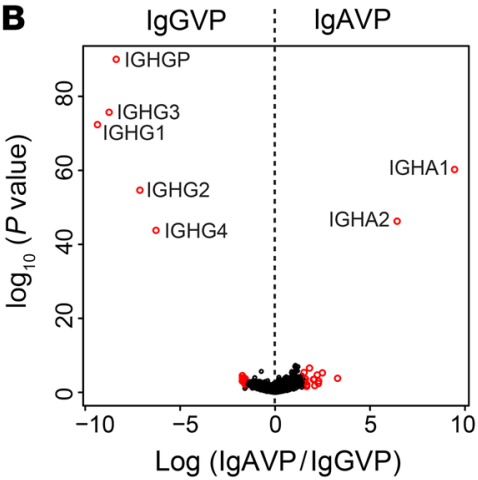

C

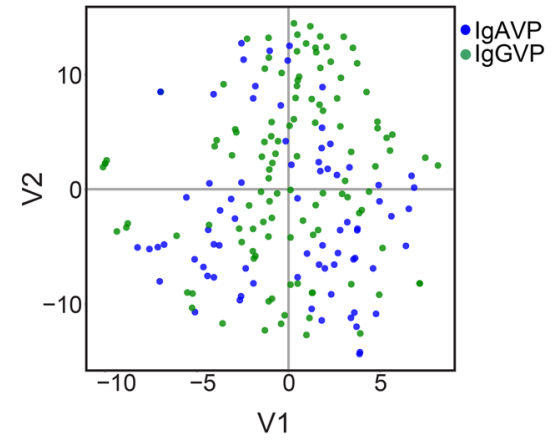

Figure 3. BCR constant domain transcripts are sufficient to cause clustering by isotype. (A) tSNE projection of vaccine-positive cells only ( $n=190)$. (B) Volcano plot between IgGVP and IgAVP cells. Genes with fold change less than or equal to 1.5 with a Benjamini-Hochberg adjusted $P \leq 0.05$ are colored red. (C) tSNE projection of vaccine-positive cells $(n=190)$ after lg constant genes are excluded.

tions increase (Supplemental Figure 5D). The latter improvement is not surprising, as diverse BCR transcripts should, in principle, increase the heterogeneity of unrelated B cells. Nevertheless, without Ig genes the average correlation within vaccine-positive clones is still significantly greater than that of unrelated vaccine-positive B cells (Figure 2D), and related B cells still cluster together (Figure 2E). Taken together, although BCR transcripts contribute to transcriptional similarity between clonal plasmablasts, the enhanced transcriptome similarity extends beyond Ig transcripts. We hypothesize this could be due to the fact that plasmablasts within a clone will have similar cellular origins and activation experiences (such as $\mathrm{T}$ cell help) that may durably imprint on the transcriptome and be passed on to clonal progeny. It is intriguing to consider that these potential similarities might drive even single pairs of related B cells to have unique transcriptional profiles.

We then hypothesized that beyond just secreting a particular class of antibody, IgG-expressing plasmablasts might be specialized for tissue immunity and plasmablasts secreting IgA specialized for mucosal immunity. As a means to screen for functional specialization of influenza-induced plasmablasts based on distinct BCR isotype, the gene expression profiles of IgAVP and IgGVP populations were compared. Visualization of these data indicated separate clustering of the 2 populations (Figure $3 \mathrm{~A}$ ). The most significant differentially expressed genes (DEGs) identified were the genes encoding the BCR constant domains themselves (Figure 3B). In fact, when each single cell was colored according to the expression level of IGHA1 or IGHG1, the clusters aligned perfectly with the assigned isotype annotation (Supplemental Figure 6 , A and B). When this analysis was repeated after exclusion of the Ig constant domain genes (Figure 3C), or all Ig genes (Supplemental Figure 6C), the observed clustering was appreciably diminished, identifying the BCR genes as sufficient to drive transcriptional separation. Notably, the tSNE projection after exclusion of all Ig genes still suggests a trend for IgG plasmablasts to cluster together and away from IgA plasmablasts (Supplemental Figure 6C). However, our sample size was insufficient to determine if the remaining differences between IgA versus IgG class-switched plasmablasts are biologically significant. As a final measure, we determined that clonal expansions consisting of both IgG and IgA clones also displayed increased transcriptional similarity, with and without Ig gene inclusion (Supplemental Figure 5E), indicating that plasmablasts arising from the same progenitor do not differentiate further after class switching to IgG or IgA. From these analyses we concluded that, besides the BCR genes themselves, peripheral blood IgG or IgA plasmablasts induced by influenza vaccination have highly similar transcriptional profiles. Additionally, the slight difference in IgAVP and IgGVP affinity likely does not account for transcriptional differences.

To determine if differences exist between antigen-induced and vaccine-negative plasmablasts, 2 analyses were performed. The first was to compare the IgAVP and IgAVN samples in isolation. The tSNE projection of the IgA cells suggests transcriptional segregation between these 2 populations (Figure 4A), which could indicate different functional attributes. A small fraction of the IgAVN cells remains intermingled within the IgAVP cells. The relative binding affinity of these cells does not correlate with their clustering and we hypothesize these are likely to be vaccine induced but specific for epitopes not assessed by our experiments. In total, 62 DEGs with an adjusted $P \leq 0.05$ and a fold change $\geq 1.5$ were identified between the IgAVP and IgAVN populations (Figure 4B). The significantly increased expression of IGHA2, VH3-23, VH3-7, and IGKC within the IgAVN population, and enrichment of IGLJ2 in the IgAVP population, are confirmed by increased total repertoire gene usage frequencies (Figure 4, B and C). To assess the impact of these genes on overall population clustering, the tSNE analysis was repeated without any Ig genes, and a similar level of clustering was identified (Figure 4D). This indicates that transcriptional differences exist beyond repertoire usage. Interestingly, the 2 most significant DEGs identified between IgAVP and IgAVN have been previously associated with differential BCR glycosylation, FUT8 and B4GALT1 (Figure 4, B and E).

Our second analysis compared the total vaccine-positive samples with the IgAVN population, after exclusion of all Ig genes. The tSNE projection confirms separation based on specificity, showing a preference for the IgGVP and IgAVP cells to cluster together and away from the IgAVN cells (Figure 5A). A larger number of DEGs were identified in this comparison, including FUT8 and B4GALT1 (Figure 5, B and C). Differential glycosylation of antibodies is known to alter immunogenicity, and both FUT8 and B4GALT1 have been 
A $\bullet \lg A V N \bullet \lg A V P$

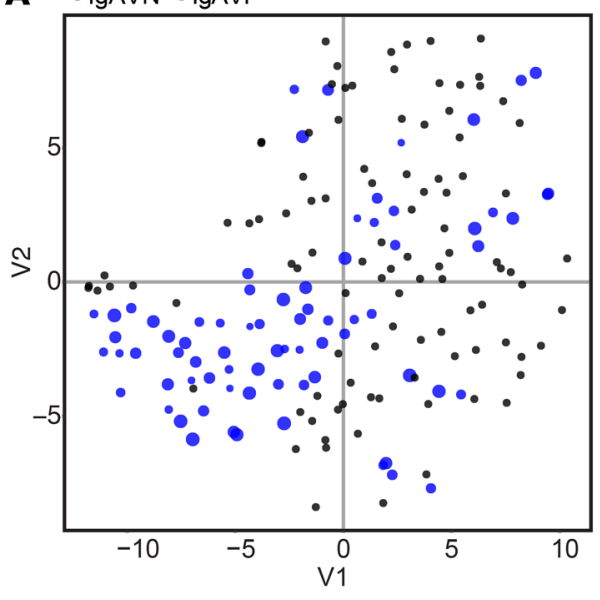

D

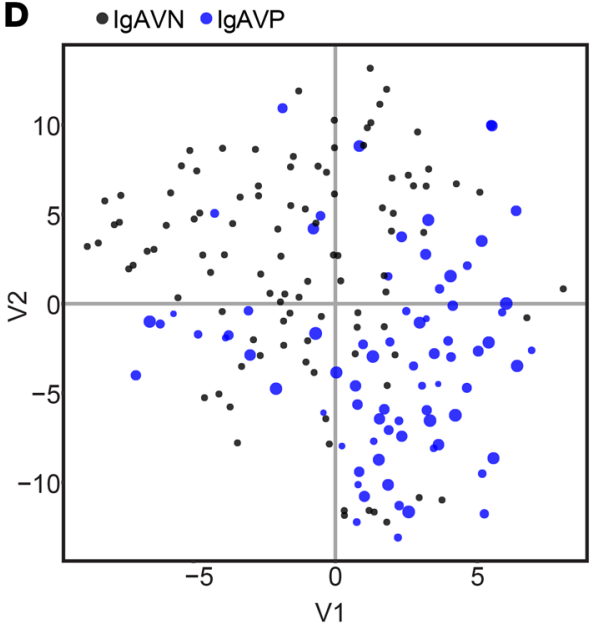

B

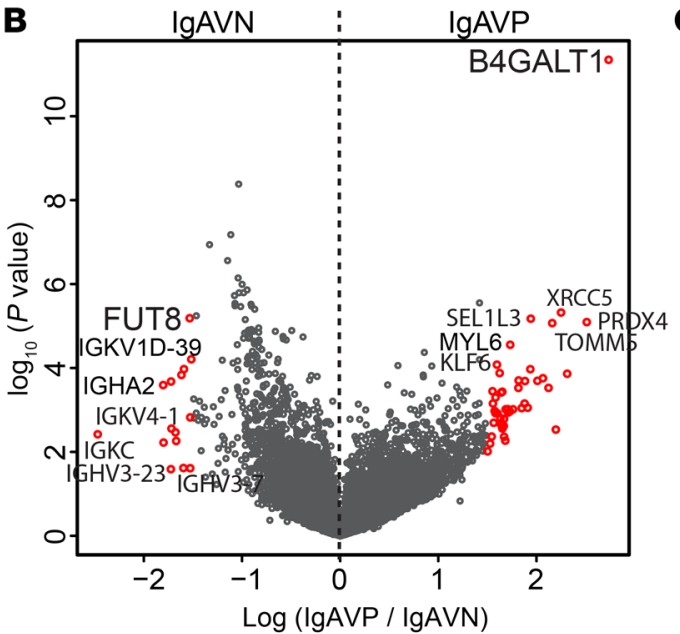

C $\frac{\lg A V P}{\square \mathrm{VH} 3 \text { family } \square \mathrm{VH} 3-7 \mathrm{VH} 3-23}$

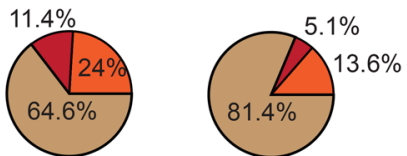

$\square$ Kappa $\square$ Lambda

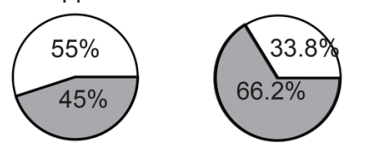

口IgA Family $\lg A 1 \quad \square \operatorname{IgA} 2$

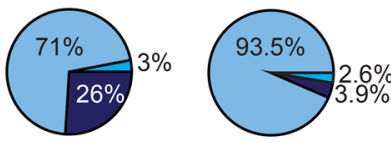

$\mathbf{E}$

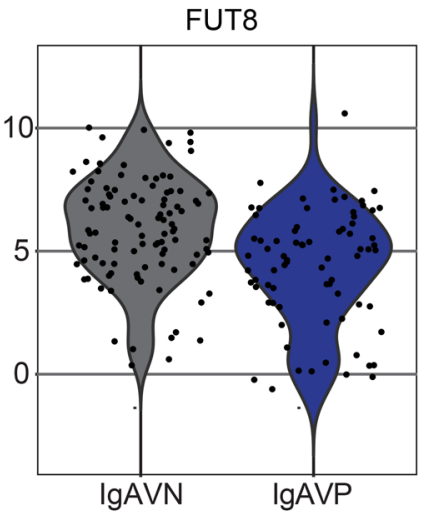

B4GALT1

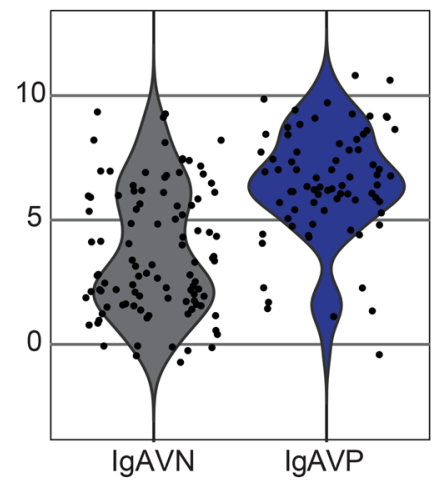

Figure 4. IgA plasmablasts cluster by their ability to bind the vaccine. (A) tSNE projection of the lgA plasmablasts ( $n=176)$, population indicated by color and relative binding affinity (area under the curve) indicated by dot size. (B) Volcano plot showing the differential expression of all genes between IgA vaccine-negative (IgAVN) and IgA vaccine-positive (IgAVP) plasmablasts. Cenes with log fold greater than or equal to 1.5 with a Benjamini-Hochberg adjusted $P \leq 0.05$ (2-sided $t$ test) are colored red. Some of the most significant genes are labeled. (C) Pie charts showing repertoire usage frequency between lgAVN and IgAVP plasmablasts. Increased usage of the lambda light chain was found within IgAVP cells $\left(P=0.0192, \chi^{2}\right)$ and increased IGHA2 usage was found within IgAVN cells $\left(P=7.45 \times 10^{-5}, \chi^{2}\right)$. (D) tSNE projection of the IgA plasmablasts repeated after the exclusion of Ig genes. (E) Violin plots of FUT8 and B4GALT1 between IgAVN and IgAVP plasmablasts, where the location on the $y$ axis shows each cell's gene expression level and the $x$ axis distribution is randomly assigned to improve spot visualization.

implicated in this process. The FUT8 enzyme is required for the addition of fucose moieties to the BCR glycans (3), whereas the B4GALT1 enzyme is necessary for the addition of galactose moieties (8). These modifications are known to shape a humoral immune response through alterations to Fc-receptor binding and complement activation (3-11). The differential expression of these enzymes between vaccine-positive and vaccine-negative cells suggests plasmablast BCR specificity directs transcriptional differences that can ascribe key effector properties to the antibodies secreted by that cell.

FOXP1 was also differentially expressed between total vaccine-binding and vaccine-negative plasmablasts $\left(P=2.06 \times 10^{-13}\right.$ and fold change of 1.49, Figure 5B and Figure 6A), as well as between the IgAVP and the IgAVN cells $\left(P=1.96 \times 10^{-5}\right.$ and fold change of -0.98 , Figure $6 \mathrm{~B})$. However, the measured fold change fell below our arbitrary 1.5 fold change requirement to be identified as a DEG of interest. The transcription factor FOXP1 is known to prevent ter- minal differentiation into antibody-secreting cells through repression of PRDM1, IFR4, and XBP1, the master transcription factors of the plasmablast fate (36). The increased expression of FOXP1 within the vaccine-negative plasmablast population may suggest upregulation of pathways designed to prevent terminal differentiation into traditional antigen-induced plasmablasts. To explore this further, we performed transcriptional cell-cycle analysis, as G1 phase arrest is a feature of terminally differentiated antibody-secreting cells $(12,13)$. This analysis revealed an increased frequency of vaccine-positive cells assigned to the G1 phase, while the IgAVN compartment displayed increased assignment to the $\mathrm{S}$ phase (Figure 6C). This trend was also detected between IgAVP and IgAVN cells (Figure 6D). These data suggest that the vaccine-negative plasmablasts may have prevented terminal differentiation through upregulation of FOXP1 to allow for continued cellular division, perhaps as a mechanism for population renewal and maintenance. 
A

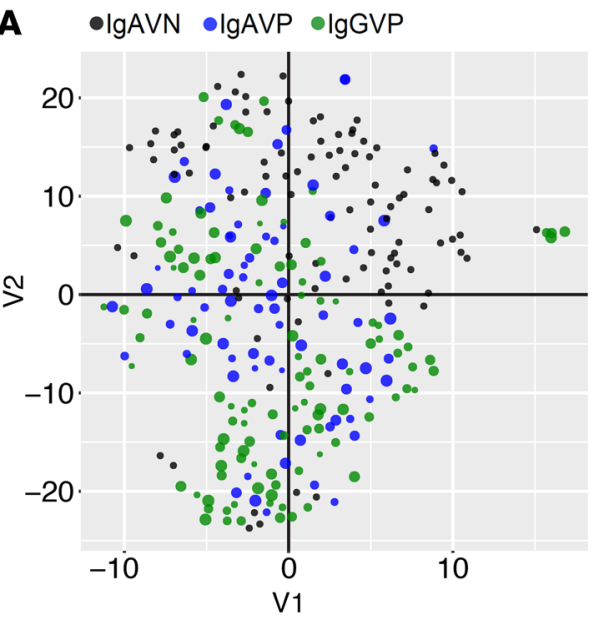

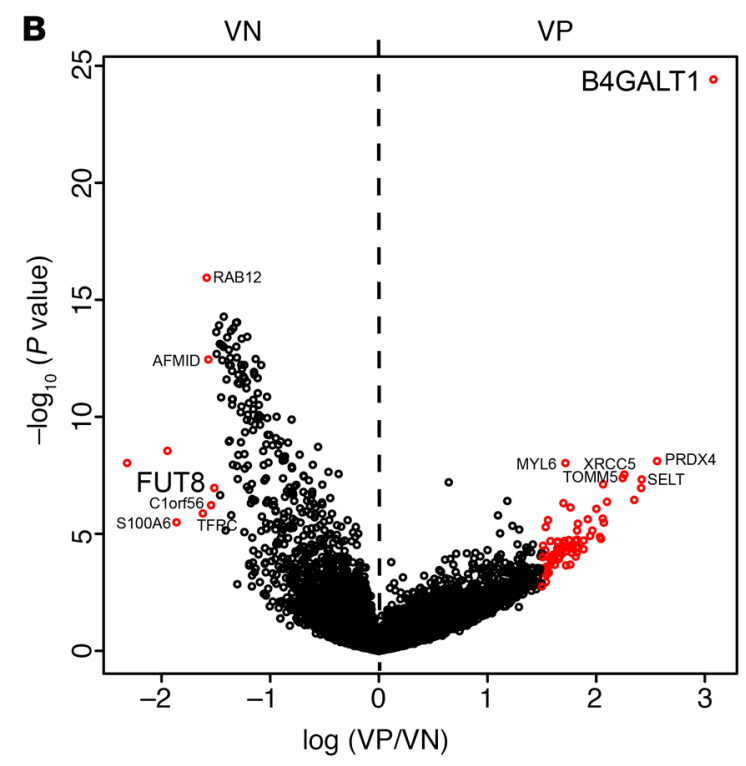

B4GALT1
C

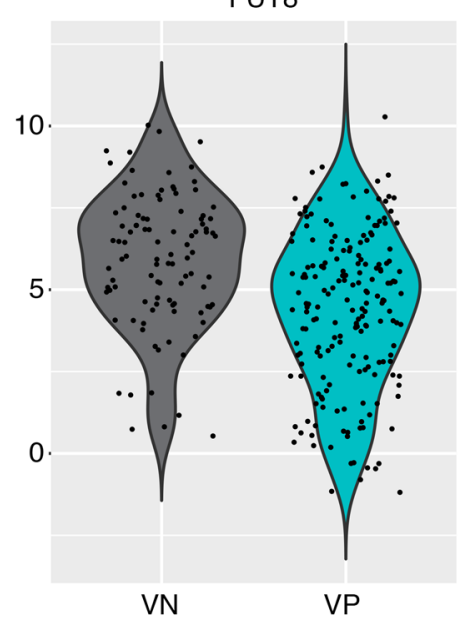

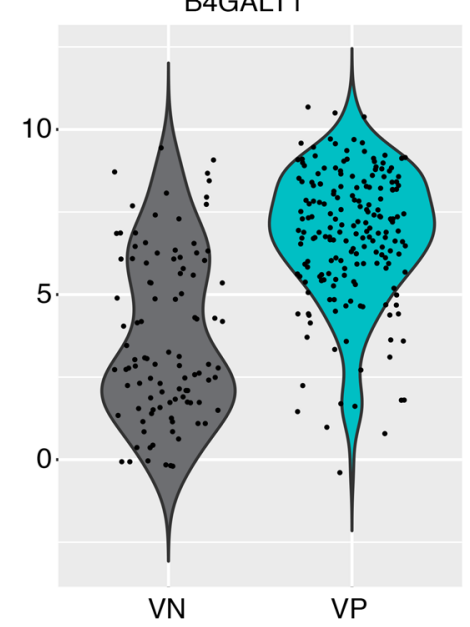

Figure 5. Total plasmablasts also cluster by their ability to bind the vaccine. (A) tSNE projection of all 3 populations after removal of $I g$ genes ( $n=291$ ) (B) Volcano plot showing DEGs between total vaccine-positive (VP) and vaccine-negative (VN) cells. (C) Violin plots showing expression of B4GALT1 and FUT8 in the VP versus VN plasmablasts.

To explore the population dynamics of IgA peripheral blood B cells, longitudinal BCR high-throughput repertoire sequencing was performed for 9 donors across 5 time points spanning 35 days. Analysis of these data identified IgA clones present 28 days prior to vaccination that are maintained within the peripheral blood over time (Supplemental Figure 7A). The average frequency of these clones present at all time points assayed ranged from $10 \%-20 \%$ of the total clonal diversity, and this increased to $20 \%-$ $55 \%$ when considering clones present for at least a month (Supplemental Figure 7B). We think this second value is a more accurate representation, as the possibility of missing a single clone at a given time point is nontrivial. An increase in total clonal diversity is evident 7 days after influenza immunization, due to expansion of many influenza-specific clones that were not detected at the earlier time points. Overall, these data indicate that peripheral blood IgA cells display a level of clonal restriction likely related to antigen selection, and that these cells maintain their presence in the peripheral blood over time. We hypothesize that the capacity for continued cell cycling detected within the IgAVN population could explain this persistence within steady-state plasmablasts.

Altogether, these data have identified transcriptional differences between vaccine-positive plasmablasts and vaccine-negative IgA plasmablasts, which support the possibility of an antigen-induced gene expression profile that may provide a novel means to identify vaccine-specific cells directly from scRNA-seq data without the need for time-exhaustive receptor generation and testing.

\section{Discussion}

In this study, we applied Spec-seq to peripheral blood plasmablasts and found that BCR transcripts are a major source of population heterogeneity. However, our data identified unexpected levels of transcriptional specialization within plasmablasts, disproving the long-standing belief that these cells are simply terminally differentiated antibody-secreting cells with limited complexity. 
A
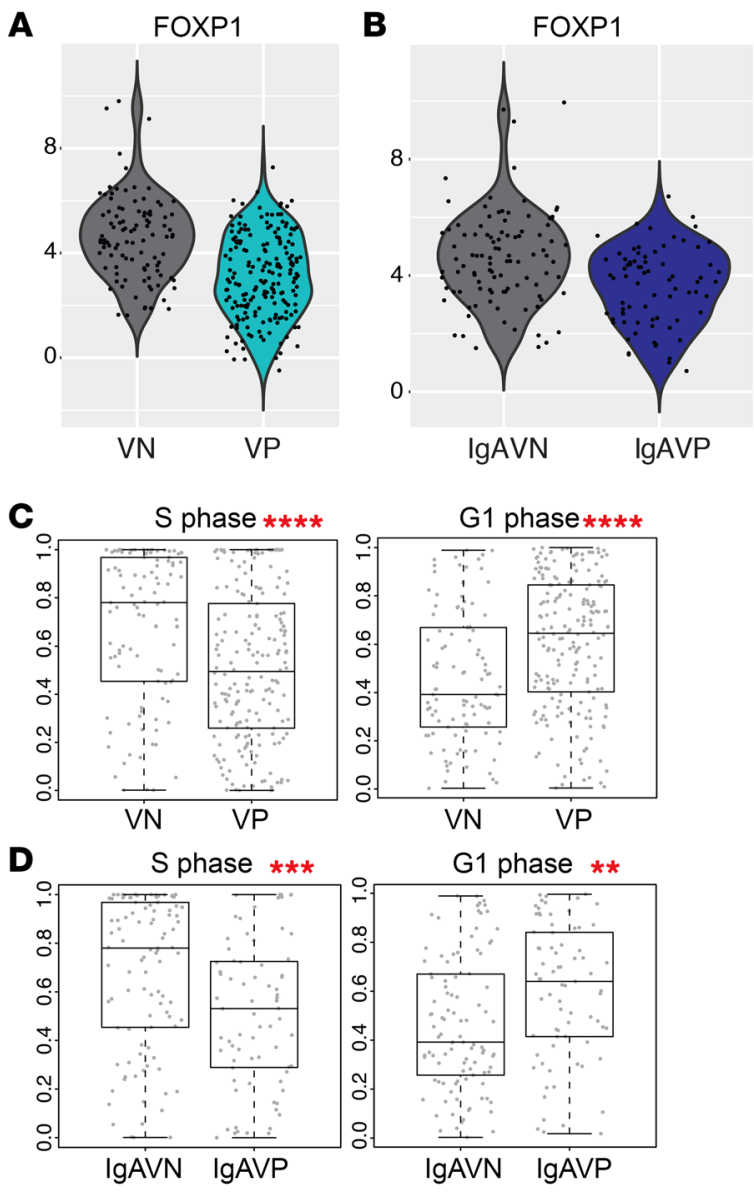

Figure 6. FOXP1 expression in IgAVN cells blocks cell-cycle arrest in G1 phase. (A) Violin plot showing FOXP1 expression between total vaccine-positive (VP) and vaccine-negative (VN) plasmablasts. (B) Violin plot for FOXP1 expression between IgAVN and IgAVP plasmablasts. (C and $\mathbf{D})$ Box plots showing differences in frequency of each assigned cellcycle stage between VP and VN plasmablasts (C) and IgAVN and IgAVP plasmablasts (D). Box plots display median cell-cycle score $\left(^{* *} P=0.0016\right.$, ${ }^{* *} P=0.00035$, and ${ }^{* * *} P<0.0001 ; 2$-sided $t$ test .

The full-length antibody sequences obtained through Specseq enabled us to perform what we believe is the first-ever analysis of the single-cell transcriptome of clonally related B cells. Unexpectedly, this analysis suggested that clones have increased transcriptional similarity beyond their shared Ig genes. We hypothesize that this could be due to transcriptional imprinting during original activation events, which are passed on to subsequent clonal progeny. The capacity for cells to inherit characteristics indicative of their clonal origin and activation history would significantly alter our understanding of heterogeneity within the humoral immune response. It will be exciting to explore this possibility further in data sets containing larger clonal expansions.

Although novel, our identification of BCR transcripts as the dominant determinant of plasmablast subpopulations is not a surprising result. These cells are highly specialized for production of antibodies, which initiates a strong unfolded protein response, the subsequent accumulation of reactive oxygen species, and eventual cell death (37). Due to this specialization, mRNA for the BCR protein will be continuously synthesized and highly overrepresented. This high expression explains why variable $\mathrm{V}(\mathrm{D}) \mathrm{J}$-C usage among B cells is responsible for a significant amount of the plasmablast transcriptional heterogeneity. Our finding that Ig constant domain gene expression is sufficient for segregation of the $\operatorname{Ig} G$ and $\operatorname{IgA}$ plasmablasts further emphasizes this point. Overall, our analysis found no additional significant changes in the gene expression profiles of cells expressing IgG versus IgA BCRs, and therefore suggests that prior CSR does not specifically induce long-term functional specialization within peripheral blood plasmablasts.

We found that differential expression of B4GALT1, FUT8, and FOXP1 is directly related to $\mathrm{BCR}$ recognition of the vaccine. Specifically, an increased frequency of vaccine-positive cells expresses high levels of the B4GALT1 enzyme, which is responsible for the addition of galactose moieties to a conserved glycan in the BCR Fc domain $(8,38)$. This modification increases the ability of $\operatorname{IgG}$ antibodies to facilitate complement-dependent cytotoxicity (10), while its presence on IgA antibodies enhances antibody-dependent cell-mediated cytotoxicity (ADCC) through improved binding affinity with the $\mathrm{Fc} \alpha \mathrm{RI}$ (11). This association between antibody vaccine recognition and galactose modification is supported in the literature, where galactose moieties have been reported to increase after influenza vaccination within the antigen-specific serum antibody compartment when compared with the steadystate serum profile (9). In contrast, the vaccine-negative plasmablasts displayed enhanced expression of the FUT8 and FOXP1 genes. The FUT8 enzyme mediates the addition of fucose moieties to BCR glycans (3). This Fc modification has been shown to reduce IgG affinity for the Fc $\gamma$ RIIIa, which limits antibody immunogenicity by reducing ADCC $(3,4)$. Although this modification is prevalent on steady-state antibodies of both IgG and IgA isotypes $(4,5)$, it remains unclear how it specifically alters IgA Fc-receptor binding $(6,7)$. Unfortunately, our mAb expression system does not preserve differential glycosylation patterns, but the increased expression of FUT8 suggests our IgAVN plasmablasts are producing antibodies with antiinflammatory properties.

The increased expression of FOXP1 within IgAVN plasmablasts suggests blockade of terminal ASC differentiation, and this is supported by the larger frequency of vaccine-negative plasmablasts assigned to the $\mathrm{S}$ phase of the cell cycle, indicating DNA replication and continued cellular division $(12,13)$. As the IgAVN cells are hypothesized to be steady-state sentinel cells recirculating from mucosal sites, it is plausible that proliferation is necessary to maintain peripheral blood population numbers. This possibility is further supported by our identification of steady-state IgA B cell clones that are maintained over time in the peripheral blood. Interestingly, we did not detect reliable expression of genes for mucosal homing receptors within this population (data not shown), which suggests that although peripheral blood ASCs likely have a shared origin with mucosal ASCs $(19,22,23)$, transcriptional profiles primarily reflect the current cellular niche. Together, these findings suggest that the IgAVN plasmablasts may represent a new, distinct subset of peripheral blood plasmablasts, one that performs a homeostatic role at the steady-state through production of antibodies lacking the inflammatory properties associated with antigen-induced antibodies and through proliferative renewal within this compartment. Further characterization 
of these antibodies to determine antigen specificity will be very interesting and could solidify a connection to the mucosa-localized, microbiota-regulating IgA ASCs.

Our findings have significant implications for the field of vaccine immunology, as our data suggest that vaccine-induced peripheral blood plasmablasts are transcriptionally distinct from the vaccine-negative IgA population. Therefore, although inducing a larger frequency of vaccine-positive IgA plasmablasts in the peripheral blood following vaccination is predicted to result in increased global levels of secretory IgA, the field should continue to focus on other ways of inducing localized mucosal protection (26-29). The direct characterization of mucosa-localized ASCs will likely be instrumental in this process.

Overall, Spec-seq opens new doors for the characterization of transcriptional heterogeneity in the context of antigen specificity. This is particularly important for cells of the adaptive immune system where unique somatically rearranged receptors identify cells of distinct antigen specificities, which equates to distinct effector functions. Many aspects of B cell biology are linked to BCR specificity and this approach will be pivotal for advancing the B cell field.

\section{Methods}

Human peripheral blood isolation. Fifteen healthy subjects were recruited to receive the 2014-2015 quadrivalent influenza vaccine (QIV) Fluarix (GSK) at the University of Chicago with approval by the institutional review board (protocol 09-043A). Peripheral blood (40-80 ml) was drawn 7 days after immunization. B cells were enriched from peripheral blood mononuclear cells (PBMCs) by the RosetteSep Human B cell Enrichment Kit (Stem Cell Technologies, catalog 15024), and treated and isolated with a Lymphoprep gradient (Corning, catalog 25-072). The 6 subjects with the highest vaccine-positive antibody-secreting cell frequency determined by ELISPSOT were selected for inclusion in our study.

ELISPOT. B cell-enriched PBMCs $\left(0.25 \times 10^{6}\right.$ to $1.0 \times 10^{6}$ cells) were transferred onto ELISPOT plates (Millipore, catalog MSHAN4B50). Plates were coated with goat anti-human immunoglobulin (whole IgG, IgM, and IgA, KPL, catalog 01-10-07) at $5 \mu \mathrm{g} /$ $\mathrm{ml}$ or with the total administered QIV at $10 \mu \mathrm{l} / \mathrm{ml}$ both in PBS overnight at $4^{\circ} \mathrm{C}$ and then blocked with complete media for 2 hours at $37^{\circ} \mathrm{C}$ before the cells were transferred. Plates with cells were incubated overnight at $37^{\circ} \mathrm{C}$. The plates were washed and then incubated with biotinylated IgG (Southern Biotech, catalog 2040-08) and biotinylated IgA (Southern Biotech, catalog 2050-08) in separate columns, for each coated antigen type for 2.5 hours at room temperature. After washing, streptavidin-alkaline phosphatase (Southern Biotech, catalog 7100-04) was added for 2.5 hours at room temperature and NBT/BCIP (Thermo Fisher Scientific, catalog 34032) was added to each well for spot reveal.

Cell sorting. The remaining enriched peripheral blood B cells were stained for fluorescence-activated cell sorting (FACS) on either a BD FACS Aria II or the BD FACS Aria Fusion at the University of Chicago flow cytometry core. Antibodies used included: CD19 (BioLegend, catalog 30223, clone H1B19), CD27 (BioLegend, catalog 302829, clone O323), CD38 (BioLegend, catalog 303515, clone HIT2), IgG (BD Biosciences, catalog 550931, clone G18-145), IgA (Southern Biotech, catalog 2050-02, clone G1012-YG02B), and IgM (Southern Biotech, catalog 9022-09, clone UHB). After gating on lymphocytes, doublet exclusion was performed. The $\mathrm{CD} 19^{+} \mathrm{B}$ cells were visualized to esti- mate the total $\mathrm{CD} 27^{++} \mathrm{CD} 38^{++}$plasmablast (PB) response. Samples were required to have more than a $0.5 \% \mathrm{~PB}$ response for inclusion in the study. The IgM ${ }^{-} \mathrm{CD} 19^{+} \mathrm{CD} 27^{++}$and $\mathrm{CD} 38^{++} \mathrm{PBs}$ were bulk sorted by IgG and IgA surface expression, prior to single-cell sorting into 96-well plates (BioRad) containing $4 \mu \mathrm{l}$ of the catching solution previously described (39). Plates were immediately sealed, placed on dry ice, and frozen at $-80^{\circ} \mathrm{C}$ until use. Each plate contained 41 single cells, 6 unsorted wells, and 1 well with 100 cells.

Simultaneous single-cell RNA sequencing and monoclonal antibody generation. This section outlines the modifications required to merge the 2 preexisting protocols as well our optimized modifications to the SmartSeq2 protocol (39).

Here we would also like to draw attention to a few improvements that were made between the processing of the first 2 tagmentation plates, and the later 3 plates. PW2 and PW3 were processed with the SuperScript III reverse transcriptase enzyme. PW4- 6 were processed with the Primescript reverse transcriptase enzyme and $5^{\prime}$ biotin modifications were added to all 3 oligomers: Oligo-dT, TSO, and IS-PCR (for sequences and additional information see ref. 39). We believe all effects of these modifications on the transcriptome data have been removed with experimental batch correction.

Cell isolation. The catching solution used for single-cell sorting was identical to that described in the SmartSeq2 protocol (39), except for the oligomer biotin modification mentioned above to reduce undesired primer-to-primer annealing and amplification. FACS was used to isolate the cells of interest, and every sorted plate contained 41 single cells, 6 wells with no cell, and a single 100-cell well. The 100-cell wells were processed at the same time and with the same protocol as that used for the single-cell wells.

cDNA reaction and amplification. The plate to be processed was thawed on ice while the reverse transcription (RT) mixture was made in the RNA-only hood. For Primescript reactions, the RT mixture contained 100 U Primescript (Clonetech, catalog 2680), 10 U RNase inhibitor (Clonetech, catalog 2313), 1M Betaine (Sigma Aldrich, cata$\log$ B0300), $6 \mathrm{mM} \mathrm{MgCl}_{2}$ (Life Technologies, catalog AM9530G), and $1 \mu \mathrm{M} 5^{\prime}$ biotin-TSO oligomer (IDT). The reaction volume was raised to $5.7 \mu \mathrm{l}$ with nuclease-free water. When the SuperScript III enzyme (Invitrogen, catalog 180800) was used, 5 mM DTT (Invitrogen, catalog 180800) was also included, as suggested in the SmartSeq2 protocol. The Oligo-dT reaction was run prior to the addition of the RT mixture. Then the SmartSeq2 RT-PCR reaction was used for all plates regardless of the reverse transcriptase enzyme.

Once complete, the preamplification PCR mix was assembled on a sterile bench top as previously described (39). We selected 22 preamplification PCR cycles for our PB populations of interest, and amplification was evaluated on an Agilent high-sensitivity DNA chip (catalog 5067-4626) (Supplemental Figure 1B). All PB traces obtained during the course of the experiment contained 2 sharp amplicon peaks, suggestive of extremely high expression for certain transcripts, the smaller of which is likely the BCR itself. The preamplification PCR was as follows: denature at $98^{\circ} \mathrm{C}$ for 3 minutes, then 22 cycles of $98^{\circ} \mathrm{C}$ for 20 seconds, $67^{\circ} \mathrm{C}$ for 15 seconds, and $72^{\circ} \mathrm{C}$ for 6 minutes, followed by $72^{\circ} \mathrm{C}$ for 5 minutes before a hold at $4^{\circ} \mathrm{C}$.

After bead-based PCR purification (39), $1 \mu \mathrm{l}$ cDNA from each cell was used in 3 separate PCRs designed to amplify the heavy, kappa, and lambda chains of the $\mathrm{BCR}$. The remaining cDNA was stored at $-80^{\circ} \mathrm{C}$ until next generation sequencing library preparation. 
Antibody PCR amplification and cloning. Antibody cloning of samples collected in 2014-2015 (PW2 and PW3) was performed as previously described by Smith et al. (16), whereas the samples collected in 2015-2016 (PW3-5) followed the revised protocol described in Ho et al. (40). No differences in antibody binding were observed with the 2 different cloning approaches (40).

Briefly, both approaches utilized separate sets of PCR to amplify the heavy chain, the kappa chain, and the lambda chain. The first PCR used a cocktail of primers designed to bind to all possible $\mathrm{V}$ gene and constant domains utilized by the respective antibody chain. The cocktail of primers used in the second PCR are nested inside the first primer binding sites to allow for additional amplification of the generated PCR product. Sanger sequencing identified the specific V(D)J or VJ combination used in each antibody chain. This information guided the primers for the cloning PCR, which incorporates either the necessary restriction digest sites for vector ligation (16) or the required sequence overhang for Gibson Assembly (40). Regardless of the cloning method used, each antibody heavy chain $\mathrm{V}(\mathrm{D}) \mathrm{J}$ was inserted into the same IgG1 backbone within the AbVec expression vector, whereas each light chain VJ portion was inserted into its respective kappa or lambda AbVec expression vector. All details of this approach are clearly outlined in the referenced papers $(16,40)$.

Library generation, pooling, and sequencing. The protocol for library generation proceeded according to the SmartSeq2 protocol. For our libraries, $0.5 \mathrm{ng}$ amplified cDNA was used for the tagmentation reaction, and 12 PCR cycles were used for the indexing PCR. After the final round of bead-based PCR purification, the Agilent bioanalyzer high-sensitivity DNA chip was used to measure fragment size and concentration (Supplemental Figure 1C). Equal volumes of all libraries were then pooled into a single tube at $3 \mathrm{nM}$ each and underwent paired-end 50 base pair sequencing in 2 lanes on the Illumina HiSeq2500 in the University of Chicago's Genomics Facility.

Antibody vector transfection. Antibody vector pairs (heavy and light chains) were then transfected into A293T cells (ATCC, catalog CRL-11268) for human monoclonal antibody production, as previously described (16). This protocol was modified slightly to allow for small volume, higher-throughput transfections. This involved coating 24 -well tissue culture plates with $2.5 \times 10^{5} 293$-A cells in $1 \mathrm{ml}$ cell media 12-18 hours before transfection. For transfection, 360 ng of each vector was mixed in $200 \mu$ l plain DMEM (Invitrogen, catalog 11995081) with $4 \mu \mathrm{l}$ polyethylenimine (Polysciences, catalog 23966-1) at $1 \mathrm{mg} /$ $\mathrm{ml}$, incubated at room temperature for 15 minutes prior to addition to the appropriate well. During the incubation period, $500 \mu \mathrm{l}$ media was removed from each plate well. After 8-16 hours, the transfection mixture was removed and replaced with protein-free hybridoma media (PFHMII; Invitrogen, catalog 12040-077). The supernatant was collected 4-5 days later, centrifuged for 10 minutes at $1,800 \mathrm{~g}$ to remove cell debris, and then used directly in screening ELISAs.

Screening ELISA. To quantify supernatant IgG content, ELISA plates (Thermo Fisher Scientific, catalog 3369) were coated with anti-human IgG (Jackson ImmunoResearch, catalog 109-1006-098) at $2 \mu \mathrm{g} / \mathrm{ml}$ in carbonate buffer. To quantify antigen binding, plates were coated with a 1:100 dilution of the influenza vaccine (Fluarix QIV, GSK) in PBS. After $12-24$ hours at $4^{\circ} \mathrm{C}$, the plates were washed and blocked with $20 \%$ FBS for 1 hour at $37^{\circ} \mathrm{C}$. A 1:25 supernatant dilution was the starting concentration for the anti-IgG plates, whereas the supernatant was used at full concentration on the influenza antigen plates. In both situations the starting solution undergoes serial dilutions down the plate to obtain a binding curve. For the anti-IgG plate, the positive control was purified human IgG (Thermo Fisher Scientific, catalog 31154) at a concentration of $250 \mathrm{ng} / \mathrm{ml}$. For the influenza vaccine-coated plates, the well-characterized CR9114 antibody (41) was used at a concentration of $10 \mu \mathrm{g} /$ ml. A horseradish peroxidase-conjugated anti-human IgG Fc-specific antibody (Mabtech, catalog 3820-4-250) was used for detection and the plates were developed with Super Aquablue ELISA Substrate (eBiosciences, catalog 00-4203-58). Absorbance was measured at a wavelength of $405 \mathrm{~nm}$ on a microplate spectrophotometer (BioRad). If a sample had an OD (minus background) greater than 0.5 on the anti-IgG plate once the positive control reached 1.0, it was considered a successful transfection. The samples on the influenza plates must also have had an OD greater than 0.5 when the positive control OD reached 3.0. The requirement for an antigen binding OD greater than 0.5 was based on previous work in our lab, where 0.5 was identified as 2 standard deviations above the mean absorbance of antibodies from naive B cells (42). Experimental replicates were performed for each antibody to confirm the results. Viral binding of mAbs was confirmed by ELISA. ELISAs were coated at 8 hemagglutination units (HAU) with either A/California/7/2009 H1N1, A/Texas/50/2012 H3N2, B/Massachusetts/2/2012 (B-Yamagata lineage), or B/Brisbane/60/2008 (B-Victoria lineage), and $\mathrm{mAbs}$ were added to each plate at $10 \mu \mathrm{g} / \mathrm{ml}$, diluted 3 -fold, and developed as described above. Area under the curve was calculated using prism with the limit of detection of $1.112 \times 10^{-8}$.

Analyzing the isotype composition of clones from high-throughput sequence data. Clonal expansions containing both IgG and IgA plasmablasts were investigated using high-throughput plasmablast sequences isolated from 3 individuals 7 days after vaccination with the 2010-2011 trivalent influenza vaccine (43). Partis (44) was used to cluster BCR sequences into clonal expansions and infer each clone's naive ancestral sequence. Briefly, Partis fits hidden Markov models to infer the most likely combination of $\mathrm{V}, \mathrm{D}$, and J genes for individual BCR sequences and compares model fit across different partitions of the data into clones to determine the most likely partition. Only productive sequences (in frame, without stop codons) for which Partis was able to infer the germline sequence were used for further analyses. Sequence isotypes were determined by using BLASTN against a custom database of constant region sequences obtained from the National Center for Biotechnology Information (NCBI). Isotypes were assigned based on the best-matching constant region sequence, provided the match had an E-value less than $10^{-2}$. Using an E-value of $10^{-3}$ produced nearly identical results (not shown). Sequences with the same E-value for different constant region sequences were considered to have an undetermined isotype. Matches involving the reverse complement of the query or reference sequences were disregarded. The frequency of clonal expansions with both IgG and IgA was calculated by dividing the number of such clones by the number of clones with more than 1 productive sequence. The maximum-likelihood tree for a representative clone was inferred from an alignment of the $\mathrm{V}, \mathrm{D}$, and J gene regions under a general time-reversible model with gamma-distributed across-site rate variation and empirical nucleotide frequencies. The alignment and the phylogenetic inference were performed using MACSE (45) and RaxML (46), respectively.

IgA repertoire sequencing and analysis. cDNA was generated from RNA extracted from PBMCs and the BCR was amplified in 3 separate PCRs pairing an IgA, IgG, or IgM reverse primer with a cocktail of V-gene primers to ensure adequate amplification of each popula- 
tion (47). The appropriately sized PCR product was gel purified before indexed libraries were generated for sequencing (New England Biolabs, E7645L and E7600S). Libraries were sequenced at $2 \times 300$ bp on the Illumina MiSeq machine. Around 8,000-12,000 IgA BCR sequences were isolated from each sample and potential clones were aligned using ClustalW hierarchical clustering. Potential clones were identified as those using the same $\mathrm{V}$ and J gene, and with the same length CDR3 loop. Next, pairwise comparisons assessing mutation frequency were performed between each BCR sequence within a potential clonal group, assigning a true clonal relationship in cases where the CDR3 sequence disparities were within $10 \%$ of the mutation frequency detected within the BCR V gene. The frequency of clonal families detected across all possible combinations of time points are reported in Supplemental Figure 7, where red indicates clones detected across all time points assayed and black indicates clones only present at a single time point.

scRNA-seq data analysis. A pipeline overview is shown in Supplemental Figure 3A.

Step 1: repertoire. Sanger BCR sequences were obtained from the nested PCR and the cloning PCR. Assembled BCR contigs were generated by applying the BASIC (31) algorithm to the raw transcriptome files for all single cells. The BCR sequences from PW2 and PW3 obtained by all 3 approaches were carefully compared previously (31) to confirm the accuracy of BASIC. In general, the assembled sequences provided by BASIC had less error and often reported a slightly reduced mutation count. This could be due in part to the high number of PCR cycles required to amplify the BCR. Therefore, we considered the assembly by BASIC to be a more accurate report of the original BCR sequence and used that contig, when available, for repertoire analysis (Supplemental Tables 1 and 2). If BASIC was unable to assemble a full-length sequence, the Sanger sequence obtained from the cloning PCR was used in its place.

Clones were identified through the same usage of $\mathrm{V}$ and $\mathrm{J}$ genes, as well as highly similar CDR3 sequences. When searching for any repertoire differences between the 3 populations of interest (Supplemental Figure 4), all clonal expansions were excluded, as their inclusion could be confused with population gene preferences. However, when validating the identified DEGs, clonal expansions were not eliminated, as those cells were present in the transcriptome (Figure 4C).

Step 2: pseudoalignment and transcript quantification. Kallisto (48, 49) was used for pseudoalignment of the raw sequencing data against the human transcriptome. The -quant command (with the -bias option) was used to perform pseudoalignment of each sample's data to the human transcriptome. Expression quantification is reported as transcripts per million (TPM), which is adjusted for transcript length and library size, and facilitates direct comparison of gene expression profiles across cells.

Step 3: Gene expression data frame, transformation, quality filters, and gene filters. The data frame of gene expression was summarized by summing each gene's transcript variants prior to undergoing $\log _{2}$ $(\mathrm{TPM}+1)$ transformation. Next, all values below 0.4 , our empirically identified threshold of detection (Supplemental Figure 3B), were set to $\mathrm{O}$ and all genes whose expression was never above $\mathrm{O}$ were removed from the data set. Our first cell-quality filter excluded cells for which less than $40 \%$ of the total reads mapped to the human transcriptome (Supplemental Figure 3C). Our next cell filter excluded cells where the $\mathrm{V}, \mathrm{J}$, and constant domains did not match between at least 2 of our 3 approaches: Sanger sequencing of the nested PCR, cloning PCR, and the assembled BASIC sequence. This filter simultaneously confirmed that each sample contained only a single cell. The constant domain of each BCR sequence must also match the flow cytometry-identified isotype. Scatter plots among single cells revealed significant variability in pairwise Pearson correlation coefficients (Supplemental Figure 3, D and E), suggesting a high level of population heterogeneity. However, when the single cells were averaged and plotted against a 100-cell well sample (Supplemental Figure $3 G$ ), the correlation coefficient matched that obtained between two 100-cell wells (Supplemental Figure 3F). Therefore, the majority of the heterogeneity is likely biological, versus technical, in nature. Next, a gene filter was applied that required expression in at least $5 \%$ of the cells, reducing the data frame to 11,895 genes.

Step 4: Quantile normalization and batch correction. Quantile normalization using the preprocess core package (50) was performed within each experimental plate to reduce differences among the quantiles of cell-specific TPM counts. Next, the Limma package (51) was used to remove known batch effects associated with experimental plate and donor ID, while protecting our variables of interest - BCR isotype and vaccine recognition (Supplemental Figure 3, H-K).

Step 5: Differential gene identification and visualization. Differentially expressed genes were identified with a Student's $t$ test, where samples were either grouped by BCR isotype or BCR vaccine reactivity. We required a fold change greater than or equal to 1.5 and a Benjamini-Hochberg adjusted $P$ less than 0.05 . Visualization of the scRNA-seq data was obtained with the Rtsne package (52). Default parameters were used, except theta $=0.001$ and initial dims $=10$. The same parameters were used for all data sets.

Cyclone cell-cycle analysis. The R package Cyclone (53) was applied to the single-cell transcriptome data to assign cell-cycle phases. This package incorporates previously published gene sets associated with G1, G2M, or S phases of the eukaryotic cell cycle. The input is the Limma-adjusted gene expression data frame.

Statistics. A paired Student's $t$ test was used to assess significant differences between the cell-cycle phase assignments (Figure 6, C and D). To identify statistically significant repertoire differences, $\chi^{2}$ tests were used (Supplemental Figure 4, A-D), in addition to 1-way ANOVA (Supplemental Figure 4, E-H). To assess global transcriptional similarity within vaccine-positive and -negative cells, we performed 2 quantitative steps (Figure 2D and Supplemental Figure 5B). First, we calculated the Pearson correlation between cells within vaccine-positive clones, and between vaccine-negative cells. Second, since correlation coefficients are bounded in $[-1,+1]$, we performed a Fisher's $Z$ transformation $[\operatorname{arctanh}(\mathrm{p})]$ on the coefficients and subjected the transformed values to a 1-sided, 2-sample Student's $t$ test. Specifically, we tested if the average correlation between clonally related cells is significantly greater than nonclonally related cells in various instances.

Data and code availability. Scripts used to generate the data in this paper can be accessed at http://ttic.uchicago.edu/ aakhan/specseq. The code for the analyses of clones from high-throughput sequence data is available at https://github.com/cobeylab/isotype_phylogenetics. All sequencing data are accessible in the Gene Expression Omnibus database under accession number GSE11650.

Study approval. Written informed consent was received from all subjects recruited at the University of Chicago for this study, with approval of the Institutional Review Board (protocol 09-043A). 


\section{Author contributions}

KEN carried out the majority of experiments and data analysis. JJG, NYZ, MET, and NJH generated monoclonal antibodies, and JJG, MET, and NJH performed ELISAs and analyzed data. $\mathrm{MH}$ performed antibody cloning. JL assisted in computational analysis of scRNAseq data. MCV, KK, and SC performed clonal analysis of the high-throughput repertoire sequencing data for Figure 2. KTR coordinated donor recruitment. NYZ, MC, and BP provided or processed samples for the IgA high-throughput repertoire sequencing studies. KTR, DS, and CLD assisted with sample collection and experiments. $\mathrm{CH}$ provided key guidance and experimental assistance. JJG, CH, CLD, and DS made significant contributions to manuscript editing. AAK directed the computational analyses. PCW conceived of the project, directed all experiments, and performed clonal analysis of high-throughput IgA repertoire sequencing studies. KEN, AAK, and PCW wrote the manuscript.

\section{Acknowledgments}

The authors thank Yunping Huang and Sarah F. Andrews for assistance with experiments, and the University of Chicago's
Flow Cytometry Facility, Comprehensive Cancer Center DNA Sequencing and Genotyping Facility, and Genomics Facility. Computational analyses were completed in part with resources provided by the University of Chicago Research Computing Center. This project was funded in part with federal funds from the National Institute of Allergy and Infectious Disease, National Institutes of Health grants U19AI082724 (to PCW), P01AI097092 (to PCW), U19AI109946 (to PCW), U19AI057266 (to PCW), HHSN272201400005C (to PCW), P30CA014599 (Genomics Facility), T32GM007281 (to DS), and T32AI007090 (to KEN). MCV and SC were supported by a Complex Systems Scholar Award from the James S. McDonnell Foundation (to SC). KK was supported by Centers of Excellence for Influenza Research and Surveillance grant HHSN272201400005C (to SC).

Address correspondence to: Patrick C. Wilson, Jules F. Knapp Building, 924 East 57th Street, R414, Chicago, Illinois 60637, USA. Phone: 773.702.9009; Email: wilsonp@uchicago.edu. Or to: Aly A. Khan, 6045 South Kenwood Avenue, Chicago, Illinois 60637, USA. Phone: 773.834.2500; Email: aakhan@ttic.edu.
1. Plotkin SA. Correlates of protection induced by vaccination. Clin Vaccine Immunol. 2010;17(7):1055-1065

2. Greenwood B. The contribution of vaccination to global health: past, present and future. Philos Trans R Soc Lond, B, Biol Sci. 2014;369(1645):20130433.

3. Yamane-Ohnuki N, et al. Establishment of FUT8 knockout Chinese hamster ovary cells: an ideal host cell line for producing completely defucosylated antibodies with enhanced antibodydependent cellular cytotoxicity. Biotechnol Bioeng. 2004;87(5):614-622.

4. Shields RL, et al. Lack of fucose on human IgG1 N-linked oligosaccharide improves binding to human Fcgamma RIII and antibody-dependent cellular toxicity. J Biol Chem. 2002;277(30):26733-26740.

5. Gomes MM, Wall SB, Takahashi K, Novak J, Renfrow MB, Herr AB. Analysis of IgA1 N-glycosylation and its contribution to FcalphaRI binding. Biochemistry. 2008;47(43):11285-11299.

6. Woof JM, Mestecky J. Mucosal immunoglobulins. Immunol Rev. 2005;206:64-82.

7. Shade KTC, Anthony RM. Antibody glycosylation and inflammation. Antibodies. 2013;2(3):392-414.

8. Keusch J, Lydyard PM, Delves PJ. The effect on IgG glycosylation of altering beta1, 4-galactosyltransferase-1 activity in B cells. Glycobiology. 1998;8(12):1215-1220.

9. Selman $\mathrm{MH}$, et al. Changes in antigen-specific IgG1 Fc N-glycosylation upon influenza and tetanus vaccination. Mol Cell Proteomics. 2012;11(4):M111.014563.

10. Hodoniczky J, Zheng YZ, James DC. Control of recombinant monoclonal antibody effector functions by $\mathrm{Fc} \mathrm{N}$-glycan remodeling in vitro. Biotechnol Prog. 2005;21(6):1644-1652.

11. Basset C, et al. Glycosylation of immunoglobulin A influences its receptor binding. Scand J Immunol.1999;50(6):572-579.
12. Chen-Kiang S. Cell-cycle control of plasma cell differentiation and tumorigenesis. Immunol Rev. 2003;194:39-47.

13. Ezhevsky SA, Toyoshima H, Hunter T, Scott DW. Role of cyclin A and p27 in anti-IgM induced G1 growth arrest of murine B-cell lymphomas. $\mathrm{Mol}$ Biol Cell. 1996;7(4):553-564.

14. Wrammert J, et al. Rapid cloning of high-affinity human monoclonal antibodies against influenza virus. Nature. 2008;453(7195):667-671.

15. Wrammert J, et al. Broadly cross-reactive antibodies dominate the human $\mathrm{B}$ cell response against 2009 pandemic H1N1 influenza virus infection. JExp Med. 2011;208(1):181-193.

16. Smith K, et al. Rapid generation of fully human monoclonal antibodies specific to a vaccinating antigen. Nat Protoc. 2009;4(3):372-384.

17. Wilson PC, Andrews SF. Tools to therapeutically harness the human antibody response. Nat Rev Immunol. 2012;12(10):709-719.

18. Moldoveanu Z, Clements ML, Prince SJ, Murphy BR, Mestecky J. Human immune responses to influenza virus vaccines administered by systemic or mucosal routes. Vaccine. 1995;13(11):1006-1012.

19. Mei HE, et al. Blood-borne human plasma cells in steady state are derived from mucosal immune responses. Blood. 2009;113(11):2461-2469.

20. Brüggemann $M$, et al. Comparison of the effector functions of human immunoglobulins using a matched set of chimeric antibodies. J Exp Med. 1987;166(5):1351-1361.

21. Kerr MA. The structure and function of human IgA. Biochem J. 1990;271(2):285-296.

22. Pabst $\mathrm{O}$. New concepts in the generation and functions of IgA. Nat Rev Immunol. 2012;12(12):821-832.

23. Iversen $\mathrm{R}$, et al. Strong clonal relatedness between serum and gut IgA despite different plasma cell origins. Cell Rep. 2017;20(10):2357-2367.

24. Neutra MR, Kozlowski PA. Mucosal vaccines: the promise and the challenge. Nat Rev Immunol. 2006;6(2):148-158.
25. Rose MA. Mucosal immunization in perspective. Hum Vaccin Immunother. 2014;10(7):2115-2117.

26. Wang BZ, Xu R, Quan FS, Kang SM, Wang L, Compans RW. Intranasal immunization with influenza VLPs incorporating membraneanchored flagellin induces strong heterosubtypic protection. PLOS ONE. 2010;5(11):e13972.

27. Ichinohe $\mathrm{T}$, et al. Cross-protection against H5N1 influenza virus infection is afforded by intranasal inoculation with seasonal trivalent inactivated influenza vaccine. J Infect Dis. 2007;196(9):1313-1320.

28. Sundararajan A, et al. Robust mucosal-homing antibody-secreting B cell responses induced by intramuscular administration of adjuvanted bivalent human norovirus-like particle vaccine. Vaccine. 2015;33(4):568-576.

29. van Riet E, Ainai A, Suzuki T, Hasegawa H. Mucosal IgA responses in influenza virus infections; thoughts for vaccine design. Vaccine. 2012;30(40):5893-5900.

30. Masayuki K. B-cell selection in germinal centers elicited by complex antigens. J Immunol. 2016;196(1 Suppl):133.10.

31. Canzar S, Neu KE, Tang Q, Wilson PC, Khan AA. BASIC: BCR assembly from single cells. Bioinformatics. 2017;33(3):425-427.

32. Maaten $L$ van der, Hinton G. Visualizing data using t-SNE. J Mach Learn Res. 2008;9:2579-2605.

33. Spencer J, Sollid LM. The human intestinal B-cell response. Mucosal Immunol. 2016;9(5):1113-1124.

34. Horns F, et al. Lineage tracing of human B cells reveals the in vivo landscape of human antibody class switching. Elife. 2016;5:e16578.

35. Benckert J, et al. The majority of intestinal IgA+ and IgG+ plasmablasts in the human gut are antigen-specific. J Clin Invest. 2011;121(5):1946-1955.

36. van Keimpema M, et al. The forkhead transcription factor FOXP1 represses human plasma cell differentiation. Blood. 2015;126(18):2098-2109.

37. Shaffer AL, et al. XBP1, downstream of Blimp-1, expands the secretory apparatus and other 
organelles, and increases protein synthesis in plasma cell differentiation. Immunity. 2004;21(1):81-93.

38. Hayes JM, et al. Glycosylation and Fc receptors. Curr Top Microbiol Immunol. 2014;382:165-199.

39. Picelli S, Faridani OR, Björklund AK, Winberg G, Sagasser S, Sandberg R. Full-length RNA-seq from single cells using Smart-seq2. Nat Protoc. 2014;9(1):171-181.

40. Ho IY, et al. Refined protocol for generating monoclonal antibodies from single human and murine $B$ cells. Jimmunol Methods. 2016;438:67-70.

41. Dreyfus C, et al. Highly conserved protective epitopes on influenza B viruses. Science. 2012;337(6100):1343-1348.

42. Wrammert J, et al. Broadly cross-reactive antibodies dominate the human $\mathrm{B}$ cell response against 2009 pandemic H1N1 influenza virus infection. J Exp Med.2011;208(1):181-193.

43. Lau D, et al. Low CD21 expression defines a popu- lation of recent germinal center graduates primed for plasma cell differentiation. Sci Immunol. 2017;2(7):eaai8153.

44. Ralph DK, Matsen FA. Likelihood-based inference of B cell clonal families. PLoS Comput Biol. 2016;12(10):e1005086.

45. Ranwez V, Harispe S, Delsuc F, Douzery EJ. MACSE: multiple alignment of coding SEquences accounting for frameshifts and stop codons. PLoS One. 2011;6(9):e22594.

46. Stamatakis A. RAxML version 8: a tool for phylogenetic analysis and post-analysis of large phylogenies. Bioinformatics. 2014;30(9):1312-1313.

47. DeKosky BJ, et al. High-throughput sequencing of the paired human immunoglobulin heavy and light chain repertoire. Nat Biotechnol. 2013;31(2):166-169.

48. Ntranos V, Kamath GM, Zhang JM, Pachter L, Tse DN. Fast and accurate single-cell RNA-seq analysis by clustering of transcript-compatibility counts. Genome Biol. 2016;17(1):112.

49. Bray NL, Pimentel H, Melsted P, Pachter L. Near-optimal probabilistic RNA-seq quantification. Nat Biotechnol. 2016;34(5):525-527.

50. Bolstad BM, Irizarry RA, Astrand M, Speed TP. A comparison of normalization methods for high density oligonucleotide array data based on variance and bias. Bioinformatics. 2003;19(2):185-193.

51. Ritchie ME, et al. Limma powers differential expression analyses for RNA-sequencing and microarray studies. Nucleic Acids Res. 2015;43(7):e47.

52. Krijthe J. Rtsne: R wrapper for Van der Maaten's Barnes-Hut implementation of t-distributed stochastic neighbor embedding. Cran-r. https://cran.r-project.org/web/packages/Rtsne/ README.html. Accessed October 29, 2018.

53. Scialdone $A$, et al. Computational assignment of cell-cycle stage from single-cell transcriptome data. Methods. 2015;85:54-61. 